Int. J. Odontostomat., 8(3):447-452, 2014.

\title{
Urgencias Odontológicas en la Provincia de Cautín-Chile, entre los Años 2009 a 2013
}

\author{
Dental Emergencies in the Cautín-Chile Province between 2009 to 2013
}

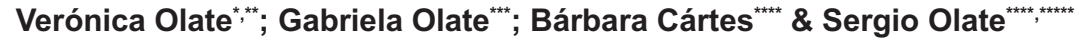

OLATE, V.; OLATE, G.; CÁRTES, B. \& OLATE, S. Urgencias odontológicas en la Provincia de Cautín - Chile, entre los años 2009 a 2013. Int. J. Odontostomat., 8(3):447-452, 2014.

RESUMEN: Las urgencias odontológicas ambulatorias comprenden un conjunto de patologías bucomaxilofaciales, de aparición súbita, de etiología múltiple, que se manifiestan principalmente por dolor agudo y que provocan una demanda espontánea de atención. El objetivo de esta investigación fue reconocer la evolución de la urgencia odontológica en los sistemas de Atención Primaria de Salud (APS) entre los años 2009 y 2013. Se realizó un estudio retrospectivo para analizar la demanda entre los años mencionados; la información se obtuvo desde los registros estadísticos mensuales de las comunas pertenecientes al Servicio de Salud Araucanía Sur (SSAS) y fueron analizados con estadística descriptiva en el programa computacional Microsoft Excel. Los resultados mostraron una tendencia a la disminución de las consultas por urgencia tanto en las garantías explicitas en salud (GES) como en las no incluidas (No GES) en los últimos años; grupos prioritarios aun son consultantes en la urgencia donde el grupo de embarazadas sufrió un aumento en las atenciones de urgencia odontológica durante los años analizados; del total de urgencias se observó una disminución de 25.000 consultas aproximadamente desde el año 2009 hasta el año 2013 con variaciones menores entre los años 2010 y 2011 . Se concluye que existe una tendencia a disminuir las consultas por urgencia GES y No GES en la población analizada aunque se deben analizar las condicionantes de grupos prioritarios en la urgencia odontológica.

PALABRAS CLAVE: urgencia odontológica, política de salud, atención primaria.

\section{INTRODUCCIÒN}

La Salud Oral es reconocida como una de las prioridades de salud del país, tanto por la percepción creciente que tiene la población sobre estas patologías como por el impacto que tiene en la salud general y en la calidad de vida (Sheiham, 2005). De este modo, la política de salud oral de Chile desde el año 2000 está orientada a la promoción y prevención, con énfasis en los grupos de riesgo, considerando actividades recuperativas en grupos priorizados mediante acciones costo efectivas basadas en la mejor evidencia disponible. La situación epidemiológica de las patologías orales en Chile muestra una alta prevalencia y severidad junto a una distribución poblacional marcada por los determinantes sociales, donde el daño se concentra en los grupos más vulnerables de nuestra sociedad (OMS, 1997).
La caries dental y la enfermedad periodontal son las enfermedades que afectan a la cavidad oral más prevalentes en el mundo (OMS, 1997). Pese a lo limitado de la información epidemiológica en Chile, se ha reportado prevalencia de caries de $70,4 \%$ y de $62,5 \%$ en población infantil de 6 y 12 años, respectivamente. La enfermedad gingival, por otra parte, también revela una alta prevalencia, afectando al $55,1 \%$ y $66,9 \%$ de los niños de 6 y 12 años (MINSAL, 2007a).

Estudios realizados por Gamonal et al. (1996) en pacientes de 35 a 44 años y 65 a 74 años, de estratos socioeconómicos bajo y medio bajo, afirman que la prevalencia de historia de caries es del $100 \%$, siendo ella la causa más frecuente de pérdida de

\footnotetext{
Programa de Magister en Salud Pública Comunitaria y Desarrollo Local, Universidad de La Frontera, Temuco, Chile.

* Departamento de Especialidades Médicas, Unidad de Medicina Familiar, Universidad de La Frontera, Temuco, Chile.

*** Programa de Odontología, Universidad Autónoma de Chile, Temuco, Chile.

***** División de Cirugía Oral y Maxilofacial, Universidad de La Frontera, Temuco, Chile.

****** Centro de Investigación en Ciencias Biomédicas, Universidad Autónoma de Chile, Temuco, Chile.
} 
dientes; el $87,38 \%$ de los pacientes ya presentaba al menos una exodoncia por caries. Resultados similares se observaron en el estudio realizado por Arteaga et al. (2009), donde se observó que la prevalencia de caries y enfermedad periodontal aumenta sostenidamente con la edad, llegando al $100 \%$ en la población adulta de 65 a 74 años.

La epidemiología asociada a la urgencia odontológica destaca que el $22 \%$ de la población general ha experimentado dolor orofacial y el $12 \%$ ha presentado dolor en alguna de sus piezas dentarias. La odontalgia es una condición con alta prevalencia en la población general y es el principal motivo de consulta (Brown, 2005), determinando un problema de salud pública debido al consiguiente ausentismo laboral, elevados costos terapéuticos y disminución de la calidad de vida de los pacientes.

En Chile, según la Encuesta Caracterización Socioeconómica Nacional (CASEN) del año 2011, un $0,7 \%$ recuerda haber tenido tratamiento por una urgencia odontológica ambulatoria en los últimos 12 meses y en comparación a la encuesta CASEN de año 2009 el número de consultas disminuyó en un $23 \%$. Ese mismo año, según la información disponible en el Departamento de Estadísticas e Información en Salud (DEIS), del total de consultas de urgencia nacional, un 20,4\% correspondió a urgencia odontológica ambulatoria (UOA).

Los sistemas de urgencia odontológica en Chile han sufrido modificaciones tanto en su esquema operacional como en la atención de usuarios durante los últimos años, principalmente debido a la inclusión de la urgencia odontológica en el tercer Régimen de Garantías Explícitas en Salud (GES), promulgado en el año 2007, evidenciando la intención del Estado de permitir el libre acceso de toda la población a la resolución de las urgencias técnicas que generan mayor ansiedad en la población por componente doloroso. Las UOA incluidas en este Régimen son: pericoronaritis, pulpitis, infecciones odontogénicas, gingivitis ulcero necrotizante, traumatismo dentoalveolar y algunas complicaciones post exodoncias como alveolitis seca, alveolitis húmeda y hemorragia post exodoncia de origen local.

En consecuencia, el objetivo de esta investigación fue describir la evolución de la urgencia odontológica y sus usuarios en la Provincia de Cautín durante los años 2009 al 2013.

\section{MATERIAL Y MÉTODO}

Se realizó un estudio retrospectivo de la atención odontológica de los usuarios de Servicios Públicos de Salud dependientes tanto de la Administración Municipal como de la Dirección de Servicio de Salud Araucanía Sur, cuyas comunas de jurisdicción'n son: Carahue, Cunco, Curarrehue, Freire, Galvarino, Gorbea, Imperial, Lautaro, Loncoche, Melipeuco, Padre Las Casas, Perquenco, Pitrufquén, Pucón, Puerto Saavedra, Teodoro Schmidt, Temuco, Vilcún, Nueva Tolten, Villarrica, Cholchol, que corresponde aproximadamente a 555.580 personas (Provincia de Cautín).

Para la obtención de datos se utilizaron los Registros Estadísticos Mensuales de Atención de Salud Odontológica, que se agrupan en los registros estadísticos anuales de todos los establecimientos de salud y comunas dependientes del Servicio de Salud Araucanía Sur, registros ingresados de forma mensual a la plataforma estadística donde se cuantifica las consultas de urgencia, actividades promocionales, preventivas y recuperativas.

La búsqueda fue realizada por un observador hasta obtener la totalidad de los registros observados, tipificando el motivo de la urgencia odontológica, las características de la misma y los diagnósticos asociados. Este trabajo se desarrolló desde la información presentada a partir del 1 de Enero del año 2009 hasta el 31 de Diciembre del año 2013. Se realizó un análisis descriptivo de la evolución de las urgencias y un análisis según los planes desarrollados por el sistema de salud mediante estadística descriptiva.

\section{RESULTADOS}

De un total de 635.905 atenciones realizadas durante el año 2009 las acciones preventivas son las que se realizan en mayor cantidad alcanzando un $39,5 \%$, dentro de esta categoría se encuentran la aplicación tópica de flúor y sellantes tanto en dentición primaria como permanente. En segundo lugar se puede observar las actividades recuperativas que corresponden al $31 \%$ de las acciones y que en el año 2013 han aumentado levemente en un $5,1 \%$. En cuarto lugar se encuentran las actividades promocionales. Tanto en las actividades promocionales, preventivas y recuperativas se observa un aumento en la cantidad de atenciones desde el año 2009 al 2013 (Tabla I). 
OLATE, V.; OLATE, G.; CÁRTES, B. \& OLATE, S. Urgencias odontológicas en la Provincia de Cautín - Chile, entre los años 2009 a 2013. Int. J. Odontostomat., 8(3):447-452, 2014.

Tabla I. Distribución de las actividades odontológicas provincia Cautín entre los años 2009 a 2013.

\begin{tabular}{lccccc}
\hline Año & $\mathbf{2 0 1 3}$ & $\mathbf{2 0 1 2}$ & $\mathbf{2 0 1 1}$ & $\mathbf{2 0 1 0}$ & $\mathbf{2 0 0 9}$ \\
\hline Promoción & 52.227 & 53.457 & 46.805 & 39.486 & 34.501 \\
Prevención & 355.171 & 345.323 & 308.056 & 279.535 & 251.178 \\
Actividades recupertativas & 207.426 & 219.585 & 206.573 & 198.744 & 197.219 \\
Total urgencia odontológica & 121.610 & 139.277 & 157.775 & 152.673 & 153.007 \\
\hline
\end{tabular}

En los registros obtenidos se objetiva una tendencia sostenida a la disminución de las UOA, pasando desde un $24 \%$ de las actividades realizadas en 2009 al $16.5 \%$ de las actividades en el 2013, destacando que a partir del 2012 este tipo de atenciones se encuentran muy por debajo de las 150.000 prestaciones que habitualmente otorgaban los servicios de salud (Fig. 1).

En el análisis por grupos se observa la disminución en las atenciones registradas como tendencia general (Tabla II). Las Figuras 1 y 2 presentan gráficamente la tendencia a la baja, donde el año 2011 se observa un leve ascenso en las consultas. En términos de análisis de urgencias comparativas entre el grupo GES y grupo No GES se observa que los primeros muestran una clara tendencia a la disminución, mientras que el grupo No GES presentó un leve ascenso en los años 2010 y 2011 que se corrige a la baja desde el año 2012.

La situación observada en grupos prioritarios como la población de 6 años, 12 años y mujeres embarazadas reporta la tendencia esta hacia la homogeneidad de consultas entre las de tipo GES y No GES, asociado principalmente al aumento de las consultas de urgencia GES y a disminuciones en la consulta No GES (figura 3). En el grupo de embarazadas, por ejemplo, se observa en el año 2009306 consultas GES y 860 consultas No GES mientras que en el año 2013 la observación es de 1187 consultas GES y 1224 consultas No GES, lo que re-

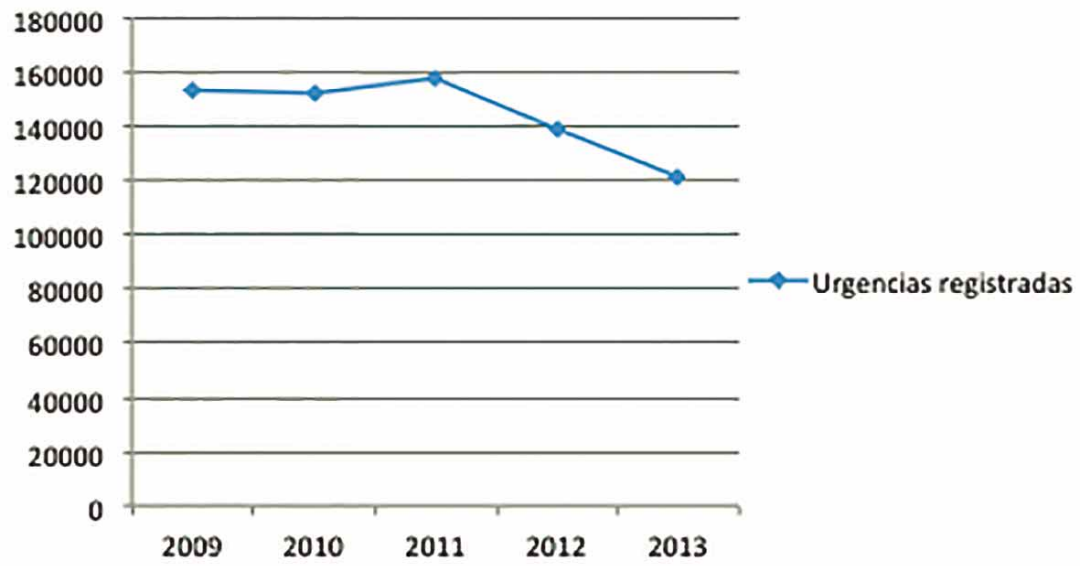

Fig. 1. Tendencia de disminución en la consulta de urgencia odontológica observada entre los años 2009 a 2013.

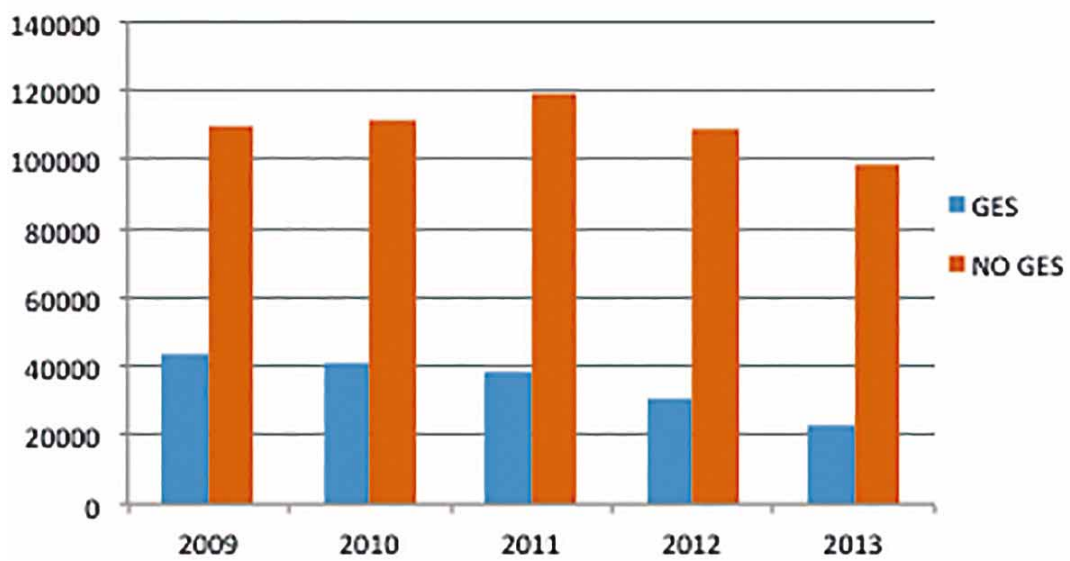

Fig. 2. Observaciones de disminución de consulta de urgencia en atención GES y No GES; GES presenta una disminución sostenida en la consulta de urgencia mientras el grupo No GES presenta variaciones menores en los años 2010 y 2011.

fleja un aumento de consultas en este grupo con la tendencia a la homogeneidad. En el grupo de 6 años y 12 años la observación muestra una disminución en las consultas por urgencia No GES; para las consultas por urgencia GES, el grupo de 6 años presenta un leve aumento mientras que el grupo de 12 años presente un leve descenso en comparación al 2009 . 


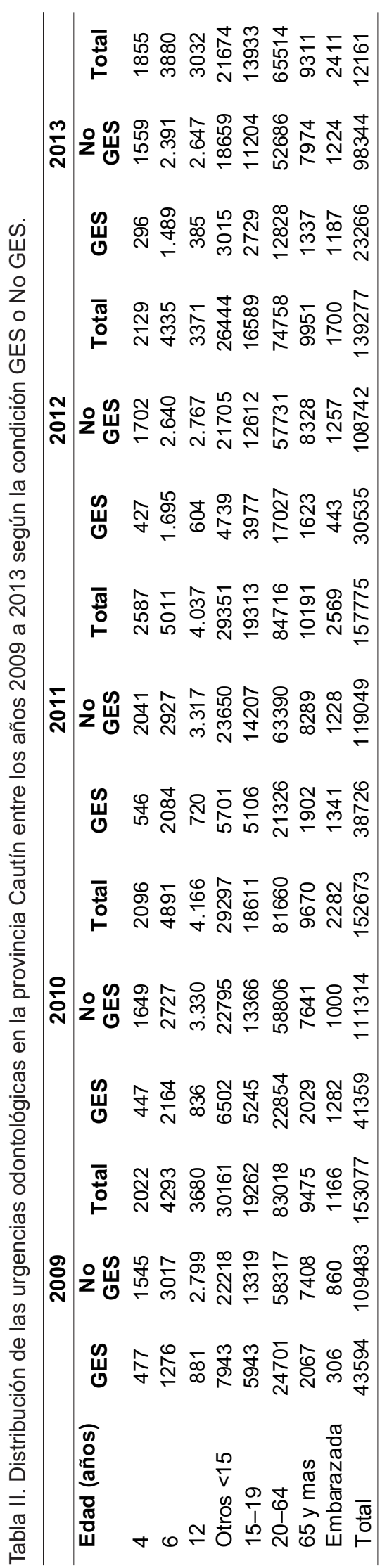

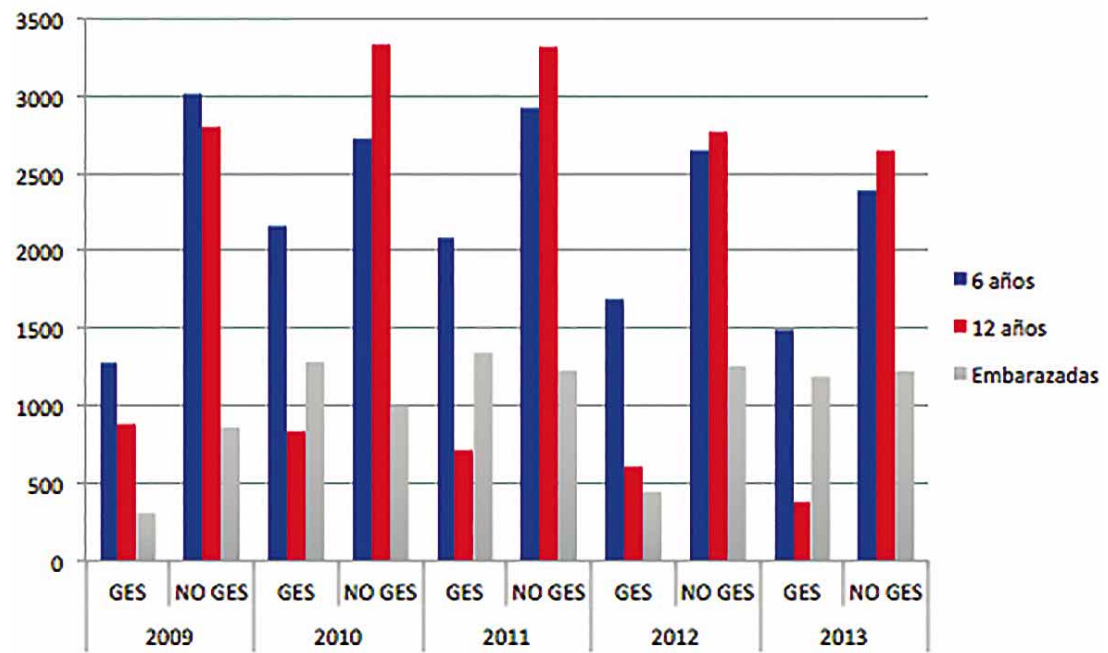

Fig. 3. Condición de consulta de urgencia dental en grupos prioritarios de 6 años, 12 años y embarazadas detectada entre los años 2009 a 2011.

\section{DISCUSIÓN}

La salud oral continúa siendo un aspecto relevante en las condiciones de salud y calidad de vida en Chile. La salud oral afecta la calidad de vida de los individuos en términos de dolor, malestar, limitación y minusvalía social y funcional. De este modo, un $37 \%$ de la población mayor de 15 años declara que su salud oral afecta su calidad de vida siempre o casi siempre, siendo los más afectados los mayores de 20 años probablemente porque su daño oral es mayor (MINSAL, 2006). Asimismo, las condiciones orales representan el 1,4\% de la carga de enfermedad medida a través de los años de vida ajustados por discapacidad (AVISA), principalmente en relación a las caries dental en menores de 45 años y edentulismo en los de 45 años y más (MINSAL, 2007b). Las patologías orales que afectan a la población son de alta prevalencia y severidad, especialmente en la población adulta, la que presenta los mayores daños por no haber accedido a programas preventivos, medidas de autocuidado y tratamientos curativos eficientes y oportunos (MINSAL, 2003), lo que determina una demanda de atención odontológica ambulatoria de urgencia constante.

Las UOA más comunes son en primera instancia el dolor dentario, con o sin infección, en adultos entre 19 y 35 años. En los niños menores de 7 años, la consulta de mayor frecuencia es el trauma dentoalveolar agudo, comúnmente causado por caídas y accidentes en bicicletas (Fodor et al., 2005). Oyarte \& Godoy (2009) presentaron un estudio de la atención clínica de urgencia realizada sobre 4.853 pacientes en el Servicio de Atención Primaria de Urgencia Odontológico del consultorio Dr. Aníbal Ariztía de la comuna de Las Condes, Santiago, entre el 1 de enero y el 31 de diciembre de 1996, donde determinaron que el $59,9 \%$ de las consultas fueron realizadas a causa de patologías derivadas de la caries dental, el $48,7 \%$ de los tratamientos efec- 
tuados fueron exodoncias, y la caries fue causa de pérdida de dientes en el $73,2 \%$ de los pacientes sometidos a exodoncias. La presente investigación se orientó a reconocer la evolución de la urgencia odontológica en el periodo 2009 a 2013 sin considerar el tipo de procedimiento realizado para el manejo de la urgencia, aunque los diagnósticos realizados también se direccionan a la caries como una de las patologías más prevalentes que motivan urgencia odontológica y pérdida dentaria (Olate et al., 2006).

Por otra parte, diversos estudios muestran que la prevalencia de enfermedades orales es mayor en personas que viven en la pobreza. La OMS reconoce que la pobreza y las desigualdades sociales juegan un papel fundamental en la presencia de enfermedades orales y en la posibilidad de recibir tratamientos. Por ejemplo, países en vías de desarrollo muestran una incidencia mucho mayor de cáncer bucofaríngeo, y el Noma, asociado a desnutrición, es casi exclusivo de África y el Sudeste asiático, las regiones más pobres del mundo (MINSAL, 2008). En Occidente, la caries y la enfermedad periodontal parecen ser un buen predictor de la pobreza en los niños y adultos, ya que el alto consumo de azúcares refinados y medidas higiénicas deficientes son más comunes en la población pobre (Petersen, 2003; Poulton et al., 2002). El nivel de enfermedad oral es mayor en la población de escasos recursos y se puede confirmar con la mayor presencia de exodoncias en la población rural (Olate et al.). Asimismo, las condiciones de vida durante la infancia determinan la salud oral del adulto (Poulton et al.).

En este sentido, la Reforma de Salud del año 2005 ha sido un avance para la salud pública de Chile, dado que en las 80 patologías hasta ahora incluidas en el régimen, la cobertura poblacional ha aumentado en números gruesos, y a pesar de que no se han cumplido a cabalidad las garantías de oportunidad prometidas, las acciones e intervenciones en salud que fueron incluidas en las guías clínicas, se han incrementado y hoy los chilenos del subsistema público pueden acceder a ellas con mayor facilidad que antes. Los resultados de los Objetivos Sanitarios para la década (2000-2010) así lo demuestran (División de Rectoría y Regulación Sanitaria, 2010). De este modo, se han diseñado diversas estrategias para disminuir la morbilidad oral y otorgar un acceso más equitativo a los servicios odontológicos, acciones que se reflejan, a modo general, en la tendencia a la disminución de las UOA, sustentadas principalmente por la disminución de las UOA GES. Excepción a esto es el grupo de embarazadas, donde la UOA GES aumento levemente en los años analizados. Causas de esta situación pueden asociarse al aumento de la población en esta condición o a las condicionantes sociales insertas en esta población (Abadia, 2006).

Las leyes del mercado y el enfoque liberal de la odontología actual hacen que la práctica odontológica se asemeje a otros bienes de consumo y sean frecuentes las negociaciones alrededor del descuento y los atrasos en los pagos (Abadia). El sistema público de salud, y particularmente, los sistemas de urgencia odontológica ambulatoria, trabajan con otra orientación, debiendo asumir condicionantes diferentes en su red de usuarios. Fernandez et al. (2011) señalan que a pesar de políticas preventivas aplicadas en Chile considerando las diferentes realidades socioeconómicas, culturales y territoriales, el mayor daño en la salud oral se observa en población de nivel socioeconómico bajo y medio. Nuestros resultados reflejan que políticas públicas que incluyen aumento del recurso humano capacitado y consecuentemente aumento de la cobertura pueden resultar en disminución de consultas por urgencia odontológica; sin embargo, es necesario mejorar las acciones de promoción y prevención para mejorar los indicadores poblacionales.

Podemos concluir que el componente preventivo y promocional debe ser un aspecto sustancial y longitudinal en los procesos de salud enfermedad y también en los regímenes garantizados. Por ello, la idea de un Plan Garantizado de aspectos preventivos incorporados en los protocolos y como parte sustantiva de los Planes Nacionales y los Objetivos de la década, darán consistencia e integralidad a las acciones de salud.

OLATE, V.; OLATE, G.; CÁRTES, B.; OLATE, S. Dental emergencies in the Cautín - Chile, Province between 2009 to 2013. Int. J. Odontostomat., 8(3):447-452, 2014.

ABSTRACT: Dental emergencies in ambulatory system included multiple oral and maxillofacial diseases with sudden onset and different etiologies; acute pain and related conditions are associated to spontaneous admission. The aim of this research was to know the evolution of the dental emergency in the public health system related to "Primary Health Attention" (HPA) between 2009 to 2013. A retrospective research was carried out on admissions between 2009 and 2013; the information was obtained from the statistical register of "Servicio de Salud Araucanía Sur"; the data was studied by descriptive statistic with Microsoft Excel software. The results showed a tendency to decrease 
the consultation by dental emergency in the GES group and the No-GES group; priority groups continue to use dental emergency services and the pregnant group showed an increase in dental emergency consult during the last few years; from the total amount of consults a reduction of approximately 25,000 was observed from 2009 to 2013 with lesser variations in the 2010 and 2011 period. It is concluded that there is a tendency of fewer GES and No-GES consultations in the analyzed population and it is necessary to understand the condition of priority groups in the dental emergency.

\section{KEY WORDS: dental emergency, health politic, primary attention.}

\section{REFERENCIAS BIBLIOGRÁFICAS}

Abadía, B. C. E. Pobreza y desigualdades sociales: un debate obligatorio en salud oral. Acta Bioeth., 12(1): 9-22, 2006.

Arteaga, O.; Urzúa, I.; Espinoza, I.; Muñoz, A. \& Mendoza, C. Prevalencia de caries y pérdida de dientes en población de 65 a 74 años de Santiago, Chile. Rev. Clin. Periodoncia Implantol. Rehabil. Oral, 2(3):161-6, 2009.

Brown, P. Caries. Mar del Plata, Editorial de la Universidad del Mar, 2005

División de Rectoría y Regulación Sanitaria. Departamento de Epidemiologi'a. Metas 2011-2020. Estrategia Nacional de Salud para el cumplimiento de los Objetivos Sanitarios de la Década 2011-2020, Santiago de Chile, Ministerio de Saluds 2010.

Encuesta Caracterización Socioeconómica Nacional (CASEN). Módulo Salud. Santiago de Chile, Ministerio de Desarrollo Social, 2011.

Fernandez, G. C.; Nuñez, F. L. \& Díaz, S. N. Determinantes de salud oral en poblacion de 12 años. Rev. Clin. Periodoncia Implantol. Rehabil. Oral, 4(3):117-21, 2011.

Fodor, A.; Navarrete, E.; Caceres, E. \& Muñoz, F. Atención de urgencia odontológica en el Hospital Urgencia Asistencia Pública. Rev. Dent. Chile, 96(3):3-6, 2005.

Gamonal, J. Prevalencia de enfermedades periodontales y de caries dental en la población de 35-44 y de 65-74 años de nivel socio-económico bajo y medio-bajo de la provincia de Santiago, región metropolitana, y determinación de los recursos humanos necesarios para su tratamiento. Rev. Fac. Odontol. Univ. Chile, 14(1):56-7, 1996.
Ministerio de Salud (MINSAL). Departamento de Salud Bucal. Norma Técnica de Urgencia Odontológica. Santiago de Chile, Ministerio de Salud, 2003.

Ministerio de Salud (MINSAL). Estudio de preferencias sociales para la definición de garantías explícitas en salud. Santiago de Chile, Ministerio de Salud, 2008.

Ministerio de Salud (MINSAL). I/ Encuesta de calidad de vida y salud. Santiago de Chile, Ministerio de Salud, 2006.

MINSAL; Soto, L.; Tapia, R.; Jara, G. \& Rodríguez, G. Diagnóstico Nacional de Salud Bucal del Niño de 6 años. Santiago de Chile, Ministerio de Salud, 2007a.

Ministerio de Salud (MINSAL). Estudio de carga de enfermedad y carga atribuible. Santiago de Chile, Ministerio de Salud, 2007b.

Olate, S.; Alister, J.P.; Soto, M.; Alveal, R.; Fuentes, J. \& Thomas, D. Extracciones e indicaciones de extracciones dentales en población rural chilena de 11 a 30 años. Av. Odontostomatol., 22(2):119-24, 2006.

Organización Mundial de la Salud (OMS). Encuestas de Salud Bucodental. Métodos Básicos. 4a ed. Ginebra, Organización Mundial de la Salud, 1997

Oyarte, R. \& Godoy, E. Estudio epidemiológico de la atención odontológica primaria de urgencia en la comuna de Las Condes, Santiago de Chile. Rev. Virtual Odontol. Ejer. Profes., 10(8), 2009. Disponible en: http:// www.odontomarketing.com/articulos/art18.htm

Petersen, P. E. The World Oral Health Report 2003. Geneva, World Health Organization, 2003.

Poulton, R.; Caspi, A.; Mine, B.; Thomson, W. M.; Taylor, A.; Sears, M. R. \& Moffitt, T. E. Association between children's experience of socioeconomic disadvantage and adult health: a life course study. Lancet, 360(9346):1640-5, 2002.

Sheiham, A. Oral health, general health and quality of life. Bull. World Health Organ., 83(9):644-5, 2005.

Dirección para Correspondencia:

Verónica Olate Morales

Unidad de Medicina Familiar

Departamento de Especialidades Médicas

Universidad de La Frontera

Claro Solar 115, Oficina 420

Temuco

CHILE

Recibido : 11-06-2014

Aceptado: 19-09-2014

Email: vero.olate@gmail.com 